\title{
Acceptance of Disability among Chinese Individuals with Spinal Cord Injuries: The Effects of Social Support and Depression
}

\author{
Jie Jiao, Mark M. Heyne, Chow S. Lam \\ Department of Psychology, Illinois Institute of Technology, Chicago, USA \\ Email: jjiao@iit.edu
}

Received June $16^{\text {th }}, 2012$; revised July $17^{\text {th }}, 2012$; accepted August $10^{\text {th }}, 2012$

\begin{abstract}
This study explored the roles of perceived social support and depression in acceptance of disability among Chinese individuals with spinal cord injuries (SCI). Design: An exploratory and cross-sectional study was conducted in an outpatient rehabilitation center in Guangzhou, China. One hundred Chinese individuals with SCI completed the Multidimensional Scale of Perceived Social Support, Center for Epidemiological Studies Short Depression Scale, and Acceptance of Disability Scale. Results: In general, there was low acceptance of disability and a high prevalence of depression among Chinese individuals with SCI. Higher acceptance of disability was associated with less depressive symptoms and higher level of perceived social support. Furthermore, depression was shown to mediate the relationship between perceived social support and acceptance of disability. Conclusion: Depression is an essential factor in the process of acceptance of disability. Cross-cultural studies are needed to facilitate a better understanding of the adjustment process following disabilities and apply culturally sensitive interventions to promote acceptance of disability.
\end{abstract}

Keywords: Acceptance of Disability; Depression; Social Support; Spinal Cord Injuries; Chinese

\section{Introduction}

Among the myriad ways that adjustment to disability has been conceptualized and studied, the concept of acceptance of disability $(\mathrm{AD})$ has received significant attention in the rehabilitation literature. At its core, $\mathrm{AD}$ is considered to be a process of perceiving one's disability as non-devaluing (Wright, 1983). To achieve this end, one must adjust his or her value system such that "actual or perceived losses from disability do not negatively affect the value of existing abilities" (Keaney \& Glueckauf, 1993: p. 200). As a psychosocial variable, AD is one of the best indicators of positive adjustment following an acquired disability (Elliott, Uswatte, Lewis, \& Palmatier, 2000) and has significant implications in vocational rehabilitation. Research has shown that AD facilitates employment and independent living (Green, Pratt, \& Grigsby, 1984) and is signifycantly associated with work status (Melamed, Groswasser, \& Stern, 1992), social integration, and overall community integration (Snead \& Davis, 2002).

There appear to be four specific value changes that characterize successful AD: enlargement of scope of values-the ability to perceive value in abilities that have not been lost as a result of the disability; subordination of physique - the ability to evaluate self-worth based on nonphysical as well as physical attributes; containment of disability effects-the ability to view the disability itself as a single characteristic of oneself, but not the only characteristic; and transformation from comparative to asset values - the ability to recognize the value of one's own unique combination of characteristics and abilities rather than making comparisons to an outside and often unattainable standard (Wright, 1983). Assessment of these four core values has been shown to be a valid method of evaluating psychosocial adjustment following acquired physical disability (Elliott,
Kurylo, \& Rivera, 2002).

The psychosocial variable that has perhaps the most consistent association with adjustment to disability in people with spinal cord injuries (SCI) is depression. Higher reported depressive symptomology has been related to lower adjustment in studies of varying sample sizes (Attawong \& Kovindha, 2005; Elliott, 1999; Krause, Brotherton, Morrisette, Newman, \& Karakostas, 2007; Martz, Livneh, Preibe, Wuermser, \& Ottomanelli, 2005). The salient relationship between depression and $\mathrm{AD}$ in those years removed from the occurrence of SCI supports the idea that $\mathrm{AD}$ encompasses more than one's immediate emotional reaction to a physical disability. AD comprises cognitive and affective elements which interact to determine one's adjustment to a chronic and disabling health condition. It may be that those with high $\mathrm{AD}$ are able to de-emphasize the importance of physical attributes and place more value on their characterological attributes, leading to a sense of empowerment and a more positive outlook on their current situation; conversely, those with low AD may be preoccupied with the negative impact of their disability, leading to a sense of helplessness and leaving them more susceptible to prolonged and/or recurrent episodes of negative mood and experience.

Social support has also been shown to be positively associated with health, well-being, and coping with a stressful event such as SCI (Chronister, Johnson, \& Berven, 2006). Some findings indicate that social support serves to buffer depression and suicidal intent, as well as enhance quality of life and life satisfaction (Beedie \& Kennedy, 2002; Kemp \& Krause, 1999). One of the characteristics of a collective culture such as China is the strong social support from family members and friends for those with disabilities (Morris \& Peng, 1994; Yang, 1981). Having a strong social network and supportive relationships is particularly desirable for Chinese people (Triandis et al., 1986), 
and should serve to alleviate depression and facilitate adjustment to disability.

A study by Belgrave (1991) found that social support was a significant predictor of $\mathrm{AD}$ among 170 African Americans with disabilities. Furthermore, Post and colleagues (1999) reported that there was a moderate to strong negative correlation between social support and depression, and a positive correlation between social support and well-being, self-esteem, adjustment, and life satisfaction among people with SCI. Beedie and Kennedy (2002) followed a group of individuals with SCI longitudinally and found that high quality of social support was associated with lower hopelessness and depression scores, with this association becoming more pronounced as rehabilitation progressed. Sherman, Devinney, and Sperling (2004) compared the impact of two types of social support, past peer-mentoring experience (PME) and current live-in partner (LIP), on adjustment after SCI. They found that PME was associated with higher occupational activity and life satisfaction, while LIP was associated with greater mobility and economic self-sufficiency. These results attest the differential impacts of social support sources. The significance of social support on subjective wellbeing and quality of life has also been reported in other studies (Hampton, 2004; Hampton \& Qin-Hilliard, 2004). In summary, people who report greater social support experience less emotional distress and better adjustment to their disabilities.

Being the most populous country in the world, China also has the largest number of people with disabilities. Unlike the US, there is no official record of the incidence of SCI in China, though the number of individuals with SCI is expected to be high and on the rise given the increase in automobile and construction accidents (Hampton, 2001). To better understand how Chinese with SCI might differ from Westerners in their adjustment to disability, it is first necessary to know whether the Chinese differ from Westerners in their manifestation of depressive symptoms, as depression is the most consistent psychosocial correlate of SCI. Previous epidemiological studies have found the lifetime prevalence of depression to be between $3 \%$ and $17 \%$ in the United States (Kessler et al., 1994), but as low as .19\% in China (Hwu, Chang, Yeh, Chang, \& Yeh, 1996). One of the proposed reasons for this large discrepancy in depression prevalence is the more somatic presentation of psychological distress in the Chinese (Draguns, 1996), leading to medical diagnoses other than depression. The overall results of studies investigating symptom reporting in Chinese with depression suggest that the tendency of the Chinese to interpret putative symptoms of depression with a somatic explanation is stronger if they are less acculturated to a Western-style culture (Parker, Chan, Tully, \& Eisenbruch, 2005; Yen, Robins, \& Lin, 2000).

Despite their significant relationship with each other, as well as with other psychosocial and disability-related factors, the effects of depression and social support on AD within the context of physical disability have not received much attention in non-Western samples. The correlates of AD in the Chinese may be somewhat different from those in U.S. samples, stemming from differing psychological reactions, differing social and physical environments, or differing cultural conceptualizations of disability. The more collectivist self-concept apparent in Eastern cultures suggests that long-term AD may be different in Chinese individuals with SCI, especially given the traumatic, chronic, and life-changing nature of this disability, and the more somatic orientation of symptom reporting among native
Chinese. For this reason, the study of the association between $\mathrm{AD}$, depression, and social support, which often being viewed as buffer to anxiety and depression, is necessary given the paucity of research done with Chinese SCI population.

The purpose of the current study was to explore the relationship between perceived social support, depression, and AD among Chinese individuals with SCI. It was hypothesized that both depression and social support would significantly predict AD. It was also hypothesized that high perceived social support would attenuate, or even nullify, the impact of depression on AD. In other words, we hypothesized that social support would serve as a mediator of the relationship between depression and AD.

\section{Method}

\section{Participants}

The current study is part of a major collaborative project between the Guangdong Provincial Vocational Rehabilitation Center and the Illinois Institute of Technology's (IIT) Rehabilitation Psychology Program. Participants were patients referred by their treating rehabilitation professionals at the rehabilitation center, located in Guangzhou, China. To be included in the study, participants had to be at least 18 months post-SCI, as indicated by medical record or self-report. The justification for this inclusion criterion was to avoid the transient impact of the traumatic event related to the SCI and assess more stable and enduring cognitive and psychological constructs. Participants had to be able to complete all instruments through faceto-face interview or phone interview.

Exclusion criteria: Exclusion criteria included concomitant traumatic brain injury (TBI), developmental disabilities, or a history of hospitalization for a psychiatric disorder, as indicated by medical record. Those with TBI or a developmental disability were excluded to ensure that all participants could understand and complete study measures. Those with a history of psychiatric hospitalization were excluded to avoid the potential influence of a previous psychiatric condition on the psychosocial variables of interest in this study.

Procedure: Two hundred twenty-five individuals were initially identified from the rehabilitation center as possible participants for the study. Based on inclusion and exclusion criteria and willingness to participate, the final sample consisted of 100 in and outpatients. The age range of the participants was from 19 to 77 (Mean $=37.68, \mathrm{SD}=11.77)$ and 37 percent of the participants had some college education. At the time of the study, more than 70 percent of the participants were married and less than half of the participants were working (full time = $31 \%$; part time $=7 \%$; home maker $=3 \%$; student $=2 \%$ ). Each participant was paid 30 RMB $(\$ 4.50)$ for their interview. Interviews took place either in person or by phone; in-person interviews lasted approximately 40 minutes and phone interviews lasted 60 - 80 minutes. All interviews were conducted by a physician or graduate research assistant trained in the administration of all measures. Prior to study participation, all participants provided written or verbal informed consent. Data were collected from January 2007 through December 2008.

\section{Measures}

Demographics and injury-related characteristics: Demographic information was collected on gender, age, marital status, education level, and work status. Work status was classified 
into one of six categories: full-time; part-time; student; retired; homemaker; unemployed. Type of injury was determined by neurologic level and the extent of lesion; injuries were classified as either tetraplegia or paraplegia based on diagnosis indicated in the medical record or self-report.

Acceptance of disability: Acceptance of Disability Scale (ADS; Linkowski, 1971) is a 50-item measure examining psychosocial adjustment among people with disabilities. The scale evaluates the degree to which people find meaning in their circumstances and maintain positive beliefs about themselves. Participants rate each item on a 6-point scale ranging from 1 (disagree very much) to 6 (agree very much), with 15 of the items reverse scored. Higher scores indicate greater acceptance of disability. The scale demonstrates high reliability, with an internal consistency coefficient of .93, and significant construct and concurrent validity (Linkowski, 1971). The Chinese version of the ADS (Wu \& Lu, 1999) has been translated and back-translated, and it has been found to be appropriate for use with a Taiwanese sample.

Depression: Center for Epidemiologic Studies Short Depression Scale (CES-D 10; Andresen, Malmgren, Carter, \& Patrick, 1994) is a 10-item self-report measure designed to assess symptoms of depression in the general population. The full-length 20-item version of the CES-D (Radloff, 1977) was designed to de-emphasize somatic complaints relative to other depression scales, and the CES-D 10 is a shorter version of this instrument. Considering the substantial medical complications related to SCI, the CES-D 10 helps to avoid the problem of over-diagnosing depression based on somatic symptoms. Participants are instructed to rate items on a 4-point scale ranging from 0 (rarely or none of the time) to 3 (all of the time), with 2 items reverse scored; a higher score indicates higher depressive symptoms. When compared to the 20 -item version of the CES$\mathrm{D}$, the CES-D 10 has demonstrated strong predictive accuracy $($ kappa $=.97)$, and test-retest reliability is comparable to that of other measures of depression $(r=.71)$ (Andresen et al., 1994). Internal consistency reliability in a sample of patients with chronic disease was .84 (Lorig, Sobel, Ritter, Laurent, \& Hobbs, 2001.) The CES-D has been widely used in various cultures, and it has specifically demonstrated construct validity within a sample of Chinese married couples (Cheung \& Bagley, 1998).

Social support: Multidimensional Scale of Perceived Social Support (MSPSS; Zimet, Dahlem, Zimet, \& Farley, 1988) is a 12-item self-report measure of the perceived level of support from three sources: family, friends, and significant other. Participants rate items on a 7-point scale ranging from 1 (very strongly disagree) to 7 (very strongly agree), with higher scores indicating a higher level of perceived social support. The MSPSS has demonstrated very good internal reliability, with Cronbach's alpha ranging from .85 - .91 (Dahlem, Zimet, \& Walker, 1991; Zimet et al., 1988). The measure has also shown construct validity, as evidenced by its negative association with measures of anxiety and depression (Zimet et al., 1988), and factorial validity, as factor analysis confirms that individuals do make distinctions between sources of social support (Dahlem et al., 1991). The Chinese version of the MSPSS has shown good internal consistency as well, ranging from .82 to .94 (Chou, 2000; Short \& Johnston, 1997; Zhang \& Norvilitis, 2002).

\section{Statistical Analysis}

To examine the relationship among perceived social support, depression, and $\mathrm{AD}$, bivariate correlation was utilized. Given that little research has been done among the Chinese population, associations of demographic variables (i.e., age, gender, education) with these psychosocial variables were also examined through correlational analysis. Hierarchical regression analysis was used to evaluate the independent contributions of depresssion and perceived social support to AD. Because this was a pilot study concerning $\mathrm{AD}$ within the Chinese population, demographic variables were entered in the first block of the regression model. Total score on the perceived social support scale (MSPSS) was entered in the second block given its crucial impact in Chinese culture. Total score on the depression scale (CES-D 10) was entered in the model last to reveal its unique contribution to $\mathrm{AD}$ with all other variables controlled. The potential mediating role of social support in the relationship between depression and $\mathrm{AD}$ was tested following the procedure recommended by Judd and Kenny (1981, as cited in Baron \& Kenny, 1986).

\section{Results}

\section{Preliminary and Correlational Analyses}

Means and standard deviations of the sample's scores on the MSPSS, CES-D 10, and ADS are provided in Table 1.

Of note, approximately 67 percent of participants met the criterion for depression using the CES-D 10 cut-off score of 10 suggested by Andresen et al. (1994). After dichotomizing the sample using the above criterion (depressed vs. non-depressed), it was found that the individuals in the depressed group reported a significantly lower level of perceived social support $(\mathrm{t}$ $=4.44, p<.01)$ and acceptance of disability $(\mathrm{t}=4.59, p<.01)$ than those in the non-depressed group. The average total score on the MSPSS was 60 and 71, respectively; the average total score on the ADS was 172 and 198, respectively.

Bivariate correlation analyses showed that there were no significant correlations between the demographic variables and the MSPSS score. Similarly, only education was significantly related to CES-D 10 score $(r=-.20, p<.05)$, with people with higher education reporting less depressive symptoms. For the ADS, all three demographic variables showed a significant bivariate correlation with the ADS score. Female gender was associated with higher $\mathrm{AD}(\mathrm{r}=.24, p<.05)$. Age was negatively related to their ADS score $(\mathrm{r}=-.22, p<.05)$, and education level was positively associated with the ADS score $(r=.34$, $p<.01)$. Of interest, for the sample as a whole, age was negatively associated with education level $(\mathrm{r}=-.33, p<.01)$. After controlling for education, the relationship between age and the ADS score became non-significant.

There were significant correlations among the ADS score, CES-D 10 score, and MSPSS score. The ADS score was negatively correlated with the CES-D 10 score $(\mathrm{r}=-.57, p<.01)$ and positively correlated with the MSPSS score $(\mathrm{r}=.28, p<.01)$. The MSPSS score was also negatively correlated with the

Table 1.

Means ans standard deviations of scores on perceived social support, depression and acceptance of disability measures for the entire sample.

\begin{tabular}{cccc}
\hline Measure & $\mathrm{M}$ & $\mathrm{SD}$ & $\mathrm{n}$ \\
\hline MSPSS & 63.81 & 12.86 & 99 \\
CES-D 10 & 11.44 & 5.44 & 99 \\
ADS & 182.18 & 29.80 & 94 \\
\hline
\end{tabular}


CES-D 10 score $(\mathrm{r}=-.45, p<.01)$, indicating that higher perceived social support is associated with less reported depressive symptoms.

\section{Regression Analysis}

Hierarchical regression analysis showed that neither age nor gender in the first block was significantly predictive of the ADS score. Education however, was found to be a significant predictor of the ADS score $(\beta=.27, \mathrm{t}=2.59, p=.01)$, indicating that people with higher education tended to have higher acceptance of their disability. In the second block, the MSPSS score was also a significant predictor of the ADS score $(\beta=.28, \mathrm{t}=$ $2.96, p=.004)$; the addition of the MSPSS score accounted for an additional $7.7 \%$ of the variance in the ADS score. The CES-D 10 score was entered in the regression model last, significantly increasing the amount of the variance explained by $16.6 \%$. The complete model accounted for almost $40 \%$ percent of the variance in the ADS score, with the CES-D 10 score being the single significant predictor $(\beta=-.48, \mathrm{t}=-4.87, p$ $<.001$ ); the MSPSS score no longer significantly predicted the ADS score $(\beta=.04, \mathrm{t}=.46, p=.65)$, and education level was only marginally significant $(\beta=.18, \mathrm{t}=1.96, p=.054)$. Our hypothesis that both depression and perceived social support would predict $\mathrm{AD}$ was partially confirmed, as the CES-D 10 score was a significant predictor of the ADS score, while the MSPSS score was not.

\section{Depression as a Mediator}

As stated above, when the CES-D 10 score was added to the regression model, the prediction of the ADS score by the MSPSS score became non-significant, suggesting that depresssion mediated the relationship between perceived social support and $\mathrm{AD}$, rather than social support mediating the relationship between depression and AD. To formally examine the potential mediating role of depressive symptoms in the relationship between perceived social support and $\mathrm{AD}$, the following procedure was utilized (Judd \& Kenny, 1981, as cited in Baron \& Kenny, 1986): regressing the mediator (i.e., the CES-D 10 score) on the independent variable (i.e., the MSPSS score) to test for a significant association; regressing the dependent variable (i.e., the ADS score) on the independent variable to test for a significant association; and regressing the dependent variable on both the independent variable and mediator to test for a significantly lower degree of association between the independent variable and the dependent variable with the mediator included in the model (Table 2).

The test results as shown in Tables 3-5 met the three criteria proposed by Baron and Kenny (1986), which are as follows: the MSPSS score significantly predicted the CES-D 10 score in the first regression equation; the MSPSS score significantly predicted the ADS score in the second equation; the CES-D 10 score significantly predicted the ADS score in the third equation, while the MSPSS score was not a significant predictor of the ADS score. Thus, it can be stated that depresssion serves as a mediator of the relationship between social support and AD. That is, with depression controlled, perceived social support no longer has a significant association with $\mathrm{AD}$. In sum, our hypothesis that perceived social support would mediate the relationship between depression and AD was not supported; instead, it was depression that mediated the relationship between perceived social support and acceptance of disability.

\section{Discussion}

The current study is a pilot study exploring the relationships among perceived social support, depression, and $\mathrm{AD}$ in a sample of Chinese individuals with spinal cord injuries. It is well established in the Western literature that acceptance of disability is positively related to social support, and negatively associated with depression. However, very few studies have examined the relationship among all three of these variables, specifically, the contributions of perceived social support and depression to $\mathrm{AD}$, and the mechanism by which they operate.

This line of research is particularly pertinent to the Chinese rehabilitation population, given the extreme challenges inherent

Table 2.

Summary of hierarchical regression analysis for variables predicting acceptance of disability.

\begin{tabular}{ccccc}
\hline & Variable & B & SEB & $\beta$ \\
\hline Step 1 & Age & -.238 & .261 & -.097 \\
& Gender & 11.411 & 7.431 & .158 \\
& Education & 5.587 & 2.157 & $.269^{*}$ \\
Step 2 & Age & -.329 & .252 & -.134 \\
& Gender & 8.671 & 7.187 & .120 \\
& Education & 5.468 & 2.069 & $.264^{*}$ \\
& MSPSS & .659 & .223 & $.281^{* *}$ \\
Step 3 & Age & -.178 & .227 & -.072 \\
& Gender & 8.810 & 6.407 & .122 \\
& Education & 3.677 & 1.881 & .177 \\
& MSPSS & .104 & .229 & .044 \\
& CES-D 10 & -2.628 & .539 & $-.484^{* *}$ \\
\hline
\end{tabular}

Note: $\mathrm{R}^{2}=.149$ for Step $1 ; \Delta \mathrm{R}^{2}=.077$ for Step $2 ; \Delta \mathrm{R}^{2}=.166$ for Step $3 ;{ }^{*} p<.05$; ${ }^{* *} p<.01$.

Table 3.

Regression analysis summary for perceived social support predicting depression.

\begin{tabular}{cccc}
\hline Predictor Variable & B & SEB & $\beta$ \\
\hline MSPSS & -.189 & .038 & $-.450^{* *}$ \\
\hline
\end{tabular}

Note: $\mathrm{R}^{2}=.203 ;{ }^{* *} p<.01$

Table 4.

Regression analysis summary for perceived social support predicting acceptance of disability.

\begin{tabular}{cccc}
\hline Predictor Variable & B & SEB & $\beta$ \\
\hline MSPSS & .663 & .236 & $.283^{* *}$ \\
\hline
\end{tabular}

Note: $\mathrm{R}^{2}=.080 ;{ }^{* *} p<.01$

Table 5.

Regression analysis summary for perceived social support and depression predicting acceptance of disability.

\begin{tabular}{cccc}
\hline Predictor Variable & B & SEB & $\beta$ \\
\hline MSPSS & -.033 & .232 & .014 \\
CES-D 10 & -3.052 & .537 & $-.562^{* *}$ \\
\hline
\end{tabular}

Note: $\mathrm{R}^{2}=.323 ;{ }^{* *} p<.01$. 
in $\mathrm{AD}$ and the increased cultural emphasis on social support. This study focused on the population of Chinese individuals with SCI because of the increased rate of injury and the traumatic impact on the individuals and their families. SCI has been studied extensively in the Western literature because of its tremendous impact on rehabilitation resources. In developing China, rehabilitation services are still limited in scope, and optimizing rehabilitation outcome using available resources is the main focus of Chinese rehabilitation professionals. Additionally, a better understanding of the adjustment process of Chinese individuals with SCI will fill an existing gap in the rehabilitation literature and help develop cultural competency among Western rehabilitation professionals.

Results showed that, as a whole, participants reported a low level of acceptance of disability; the mean ADS score in this sample was 182, whereas in a Western sample of rehabilitation clients a mean ADS score of 217 has been reported, with scores of 180 and below being considered indicative of low AD (Jointer, Lovett, \& Goodwin, 1989). Compared with their Western counterparts, this Chinese sample demonstrated a lower level of AD. In terms of depressive symptoms, the mean CES-D 10 score was 11 , with more than 60 percent of participants meeting the criterion for depression using the suggested cut-off score of 10 reported by Andresen et al. (1994). This level of depressive symptomology is much higher than that reported in the Western rehabilitation literature (Richards, Kewman, \& Pierce, 2000). Due to the physical complaints commonly reported by individuals with SCI, this study used the CES-D 10 to prevent over-diagnosing depression in this population based on somatic symptoms. Thus, we believe that this result accurately reflects the depression prevalence among Chinese individuals with SCI, as somatic symptoms have been controlled by the depression measure chosen. It should be noted that the participants in this study were at least a year-and-a-half post-injury. Based on our findings and methodology, we are confident that those met the criterion for depression were not experiencing inflated symptomology due to very recent onset of injury or a preponderance of somatic symptoms. We believe that our findings are an accurate reflection of the significantly lower level of perceived social support and acceptance of disability in those with higher depressive symptomatology.

The present study confirmed the findings reported in the Western literature that female individuals are more accepting of their disability. In addition, education was positively associated with AD. Consistent with previous research, age did not demonstrate a significant association with $\mathrm{AD}$. As for the relationship between perceived social support, depression, and AD, our results replicated existing findings in the literature (Attawang \& Kovindha, 2005; Belgrave, 1991; Perry, Nicholas, \& Middleton, 2009; Post et al., 1991): perceived social support was shown to be positively associated with $\mathrm{AD}$ and negatively associated with depression; depression and $\mathrm{AD}$ were negatively associated.

By way of hierarchical regression analysis, the prediction of AD by perceived social support and depression was examined. In the regression model, education was the single significant demographic predictor of $\mathrm{AD}$, with higher education contributing to higher AD. Perceived social support was added in the second block of the model, and depression was added in the third and final block. For the overall regression equation, depression was the single significant predictor of AD. Surprisingly, perceived social support was no longer a significant pre- dictor after depression was added into the regression model.

The non-significant role of perceived social support in the final model was unexpected, given the seeming importance of social support to well-being cited in the Chinese literature (Chan, Lee, \& Lieh-Mak, 2000). Results indicated that perceived social support did not have a direct effect on AD, nor did it impact the relationship between depression and AD. Instead, depression mediated the relationship between perceived social support and AD. In other words, depression appears to be the generative mechanism through which perceived social support influences AD. That is, depression seems to be the essential factor in the process of acceptance of disability. Due to the drastic life changes resulting from their disability, Chinese individuals with SCI are likely to be sensitive to the negative impact on their social network, which can induce substantial guilt and self-blame. Furthermore, their collectivist cultural upbringing dictates that Chinese individuals seek out and require feedback from their family and friends to establish their selfconcept. So low perceived social support can be especially detrimental. This low level of perceived social support may exacerbate already existing adjustment difficulties and make these individuals more vulnerable to developing psychological problems such as depression. The results of the present study indicate that depression is prevalent among Chinese individuals with SCI, and it has a marked impact on the process of acceptance of disability.

The most significant finding of this study is the essential role of depression in the relationship between perceived social support and acceptance of disability. Rather than being mediated by social support, depression mediates the relationship between social support and acceptance of disability, having a pervasive and direct impact throughout the adjustment process. For social support to facilitate acceptance of disability, it must operate by mitigating individuals' depressive symptoms. One explanation for this finding is that Chinese individuals with SCI are particularly vulnerable to depression as the result of their disabling condition. More than 60 percent of the participants in this study met the CES-D 10 criterion for depression (Andresen et al., 1994), and this prevalence is higher than that documented in the Western literature (Richards et al., 2000). Another possible explanation for only the indirect impact of social support may be that those with SCI need social support aside from family, friends, and significant others, as measured by the social support scale used in this study. Subsequent studies should investtigate whether support from rehabilitation professionals or people with similar conditions plays a more direct role in the adjustment process.

The current study holds important implications. Given its pervasive and direct impact on the rehabilitation process, depression should be carefully assessed and closely monitored in Chinese individuals with SCI. When working with these individuals, rehabilitation professionals should pay close attention to both presenting somatic symptoms and emotional functioning, as this population appears to be at significant risk for depression. Additionally, though social support is likely an important factor in adjustment to disability, it is not a panacea. It is often assumed that as long as individuals with SCI have support from their family and friends, they will make a smooth adjustment to their disability. This is especially believed to be true for those from a collectivist cultural background such as the Chinese. Rehabilitation professionals need to guard against the tendency to oversimplify and generalize the rehabilitation 
process of people from different cultural backgrounds. Finally, the results of the present study do not undermine the importance of social support. Instead, it suggests that the conceptualization of social support and its measurement be broadened (e.g., interaction with rehabilitation professional, interaction with people with similar condition), both in research and practice, to further examine and utilize its contribution to adjustment to disability.

\section{Limitation}

The present study has several limitations. First, we used a convenient sample, and participants had been receiving outpatient rehabilitation services which are not commonly accessible for the majority of Chinese individuals with disabilities. Also, approximately 37 percent of the participants had some college education, which is higher than the level of education reported in the general population. These factors may limit the generalizability of the current findings to the larger population of Chinese individuals with SCI. Second, ours was the first study to use the ADS scale with Chinese individuals from mainland China. Studies examining the reliability and validity of this scale for this particular population are needed. Third, as discussed previously, the social support scale used in this study focuses mainly on support from family, friends, and significant others. The scale does not include social support from other resources. For example, it does not include perceived support from professionals or people with similar conditions, which might partially account for the non-significant prediction of acceptance of disability by perceived social support. Future studies on acceptance of disability, social support, and depresssion would benefit from the inclusion of a broader range of demographic, disability-related, and psychosocial variables, such as employment status, marital status, functional limitations, and self-efficacy. The interaction among these variables could shed further light on this important rehabilitation topic.

\section{Conclusion}

In summary, the results of the present study indicate that the prevalence of depression is alarmingly high among Chinese individuals with SCI. Depression is negatively associated with perceived social support, and is predictive of lower acceptance of disability. Depression is an essential factor in the adjustment process, and it mediates the relationship between social support and acceptance of disability. Further research is needed to continue investigating the effects of depression and perceived social support on acceptance of disability in populations from different cultures. Cross-cultural studies will help rehabilitation professionals develop a better understanding of the adjustment process and apply effective and culturally sensitive intervenetions to promote acceptance of disability.

\section{REFERENCES}

Andresen, E. M., Malmgren, J. A., Carter, W. B., \& Patrick, D. L. (1994). Screening for depression in well older adults: Evaluation of a short form of the CES-D (Center for Epidemiologic Studies Depression Scale). American Journal of Preventive Medicine, 10, 77-84.

Attawong, T., \& Kovindha, A. (2005). The influencing factors of acceptance of disability in spinal cord injured patients. Nepal Journal of Neuroscience, 2, 67-70.

Baron, R. M, \& Kenny, D. A. (1986). The moderator-mediator variable distinction in social psychological research: Conceptual, strategic, and statistical considerations. Journal of Personality and Social Psychology, 51, 1173-1182. doi:10.1037/0022-3514.51.6.1173

Beedie, A., \& Kennedy, P. (2002). Quality of social support predicts hopelessness and depression post spinal cord injury. Journal of Clinical Psychology in Medical Settings, 9, 227-234. doi:10.1023/A:1016003428370

Belgrave, F. Z. (1991). Psychosocial predictors of adjustment to disability in African Americans. Journal of Rehabilitation, 57, 37-40.

Chan, R. C. K., Lee, P. W. H, \& Lieh-Mark, F. (2000). The pattern of coping in persons with spinal cord injuries. Disability and Rehabilitation, 22, 501-507. doi:10.1023/A:1016003428370

Cheung, C. K., \& Bagley, C. (1998). Validating an American scale in Hong Kong: The Center for Epidemiological Studies Depression Scale (CES-D). The Journal of Psychology, 132, 169-186. doi:10.1080/00223989809599157

Chou, K. L. (2000). Assessing Chinese adolescents' social support: The multidimensional scale of perceived social support. Personality and Individual Differences, 28, 299-307. doi:10.1016/S0191-8869(99)00098-7

Chronister, J. A., Johnson, E. K., \& Berven, N. (2006). Measuring social support in rehabilitation. Disability and Rehabilitation, 28, 7584. doi:10.1016/S0191-8869(99)00098-7

Dahlem, N. W., Zimet, G. D., \& Walker, R. (1991). The multimensional scale of perceived social support: A conformation study. Journal of Clinical Psychology, 47, 756-761.

doi:10.1002/1097-4679(199111)47:6<756::AID-JCLP2270470605>3 $0 . \mathrm{CO} ; 2-\mathrm{L}$

Draguns, J. G. (1996). Abnormal behaviour in Chinese societies: Clinical, epidemiological, and comparative studies. In M. H. Bond (Ed.), Handbook of Chinese psychology (pp. 412-428). Hong Kong: Oxford University Press.

Elliott, T. R. (1999). Social problem-solving abilities and adjustment to recent-onset spinal cord injury. Rehabilitation Psychology, 44, 315332. doi:10.1037/0090-5550.44.4.315

Elliott, T. R., Kurylo, M., \& Rivera, P. (2002). Positive growth following acquired physical disability. In C. R. Snyder \& S. J. Lopez (Eds.), Handbook of positive psychology (pp. 687-699). New York: Oxford University Press.

Elliott, T. R., Uswatte, G., Lewis, L., \& Palmatier, A. (2000). Goal instability and adjustment to physical disability. Journal of Counseling Psychology, 47, 251-265. doi:10.1037/0022-0167.47.2.251

Green, B., Pratt, C., \& Grigsby, T. (1984). Self-concept among persons with long-term spinal cord injury. Archives of Physical Medicine and Rehabilitation, 65, 751-754.

Hampton, N. Z. (2001). Disability status, perceived health, social support, self-efficacy, and quality of life among people with spinal cord injury in the People's Republic of China. International Journal of Rehabilitation Research, 24, 69-71. doi:10.1097/00004356-200103000-00010

Hampton, N. Z. (2004). Subjective well-being among people with spinal cord injuries: The role of self-efficacy, perceived social support, and perceived health. Rehabilitation Counseling Bulletin, 48, 31-37. doi: $10.1177 / 00343552040480010401$

Hampton, N. Z., \& Qin-Hilliard, D. B. (2004). Dimensions of quality of life for Chinese Adults with spinal cord injury: A qualitative study. Disability and Rehabilitation, 26, 203-212. doi:10.1080/09638280310001639704

Hwu, H. G., Chang, I. H., Yeh, E. K., Chang, C. J., \& Yeh, L. L. (1996). Major depressive disorder in Taiwan defined by the Chinese Diagnostic Interview Schedule. Journal of Nervous and Mental Disease, 184, 497-502. doi:10.1080/09638280310001639704

Joiner, J. G., Lovett, P. S., \& Goodwin, L. K. (1989). Positive assertion and acceptance among persons with disabilities. Journal of Rehabilitation, 2, 22-29.

Keaney, K. C. M. H., \& Glueckauf, R. L. (1993). Disability and value change: An overview and reanalysis of acceptance of loss theory. Rehabilitation Psychology, 38, 199-210. doi:10.1037/h0080297

Kemp, B. J, \& Krause, J. S. (1999). Depression and life satisfaction among people ageing with post-polio and spinal cord injury. Disability and Rehabilitation, 21, 241-249. 
doi: $10.1080 / 096382899297666$

Kendall, E., \& Buys, N. (1998). An integrated model of psychosocial adjustment following acquired disability. Journal of Rehabilitation, 64, 16-20.

Kessler, R. C., McGonagle, K. A., Zhao, S., Nelson, C. B., Hughes, M., Eshleman, S., Wittchen, H., Kendler, K. S. (1994). Lifetime and 12month prevalence of DSM-III-R psychiatric disorders in the United States. Archives of General Psychiatry, 51, 8-19. doi:10.1001/archpsyc.1994.03950010008002

Krause, J. S., Brotherton, S. S., Morrisette, D. C., Newman, S. D., \& Karakostas, T. E. (2007). Does pain interference mediate the relationship of independence in ambulation with depressive symptoms after spinal cord injury? Rehabilitation Psychology, 52, 162-169. doi:10.1037/0090-5550.52.2.162

Linkowski, D. (1971). A scale to measure acceptance of disability. Rehabilitation Counseling Bulletin, 14, 236-244.

Lorig K. R., Sobel, D. S., Ritter P. L., Laurent, D., \& Hobbs, M. (2001). Effects of a self- management program for patients with chronic disease. Effective Clinical Practice, 4, 256-262.

Martz, E., Livneh, H., Preibe, M., Wuermser, L. A., \& Ottomanelli, L. (2005). Predictors of psychosocial adaptation among people with spinal cord injury or disorder. Archives of Physical Medicine and Rehabilitation, 86, 1182-1192. doi:10.1016/j.apmr.2004.11.036

Melamed, S., Groswasser, Z., \& Stern, M. J. (1992). Acceptance of disability, work involvement and subjective rehabilitation status of traumatic brain-injured (TBI) patients. Brain Injury, 6, 233-243. doi:10.3109/02699059209029665

Morris, M. W., \& Peng, K. (1994). Culture and cause: American and Chinese attributions for social and physical events. Journal of Personality and Social Psychology, 67, 949-971. doi:10.1037/0022-3514.67.6.949

Parker, G., Chan, B., Tully, L., \& Eisenbruch, M. (2005). Depression in the Chinese: The impact of acculturation. Psychological Medicine, 35, 1475-1483. doi:10.1017/S0033291705005623

Perry, K. N., Nicholas, M. K., \& Middleton, J. (2009). Spinal cord injury-related pain in rehabilitation: A cross-sectional study of relationship with cognition, mood and physical function. European Journal of Pain, 13, 511-517. doi:10.1016/j.ejpain.2008.06.003

Post, M. W. M., Ros, W. J. G, \& Schrijvers, A. J. P. (1999). Impact of social support of health status and life satisfaction in people with spinal cord injury. Psychology and Health, 14, 679-695. doi:10.1080/08870449908410757
Radloff, L. S. (1977). The CES-D Scale: A self-report depression scale for research in the general population. Applied Psychological Measurement, 1, 385-401. doi:10.1177/014662167700100306

Richards, J. S., Kewman, D. G., \& Pierce, C. A. (2000). Spinal cord injury. In F. G. Frank \& T. R. Elliott (Eds.), Handbook of Rehabilitation Psychology (pp. 11-27). Washington, DC: American Psychological Association. doi:10.1037/10361-001

Sherman, J. E., DeVinney, D. J., \& Sperling, K. B. (2004). Social support and adjustment after spinal cord injury: Influence of past peermentoring experiences and current live-in partner. Rehabilitation Psychology, 49, 140-149. doi:10.1037/0090-5550.49.2.140

Short, K. H., \& Johnston, C. (1997). Stress, maternal distress, and children's adjustment following immigration: The buffering role of social support. Journal of Consulting and Clinical Psychology, 65, 494-503. doi:10.1037/0022-006X.65.3.494

Snead, S. L., \& Davis, J. R. (2002). Attitudes of individuals with acquired brain injury towards disability. Brain Injury, 16, 947-953. doi:10.1080/02699050210147211

Triandis, H. C., Bontempo, R., Betancourt, H., Bond, M., Leung, K., Brenes, A., Georgas, J., Hui, C. H., Martin, G., Setiadi, B., Sinha, J., Verma, J., Spangenberg, J., Touzard, H. \& Montmollin, G. (1986). The measurement of the etic aspects of individualism and collectivism across cultures. Australian Journal of Psychology, 38, 257-267. doi: 10.1080/00049538608259013

Wright, B. A. (1983). Physical disability: A psychosocial approach (2nd ed.). New York: Harper \& Row. doi:10.1037/10589-000

Wu, W. D., \& Lu, T. H. (1999). The Chinese translated version of acceptance of disability scale. Taipei: National Taiwan Normal University.

Yang, K. S. (1981). Social orientation and individual modernity among Chinese students in Taiwan. Journal of Social Psychology, 113, 159170. doi:10.1080/00224545.1981.9924368

Yen, S., Robins, C. J., \& Lin, N. (2000). A cross-cultural comparison of depressive symptom manifestation: China and the United States. Journal of Consulting and Clinical Psychology, 68, 993-999. doi:10.1037/0022-006X.68.6.993

Zhang, J., \& Norvilitis J. M. (2002). Measuring Chinese psychological well-being with western developed instruments. Journal of Personality Assessment, 79, 492-511. doi:10.1207/S15327752JPA7903 06

Zimet, G. D., Dahlem, N. W., Zimet, S. G., \& Farley, G. K. (1988). The Multidimensional Scale of Perceived Social Support. Journal of Personality Assessment, 52, 30-41. doi:10.1207/s15327752jpa5201_2 